\section{Schlaganfall: Ist der Erfolg der Thrombektomie abhängig vom Anästhesie-Verfahren?}

Retrospektive Daten der MRCLEAN-Studie zeigen, dass Schlaganfallpatienten, bei denen die Thrombektomie in Allgemeinanästhesie erfolgte, schlechtere klinische Ergebnisse aufweisen als Behandelte ohne Narkose.

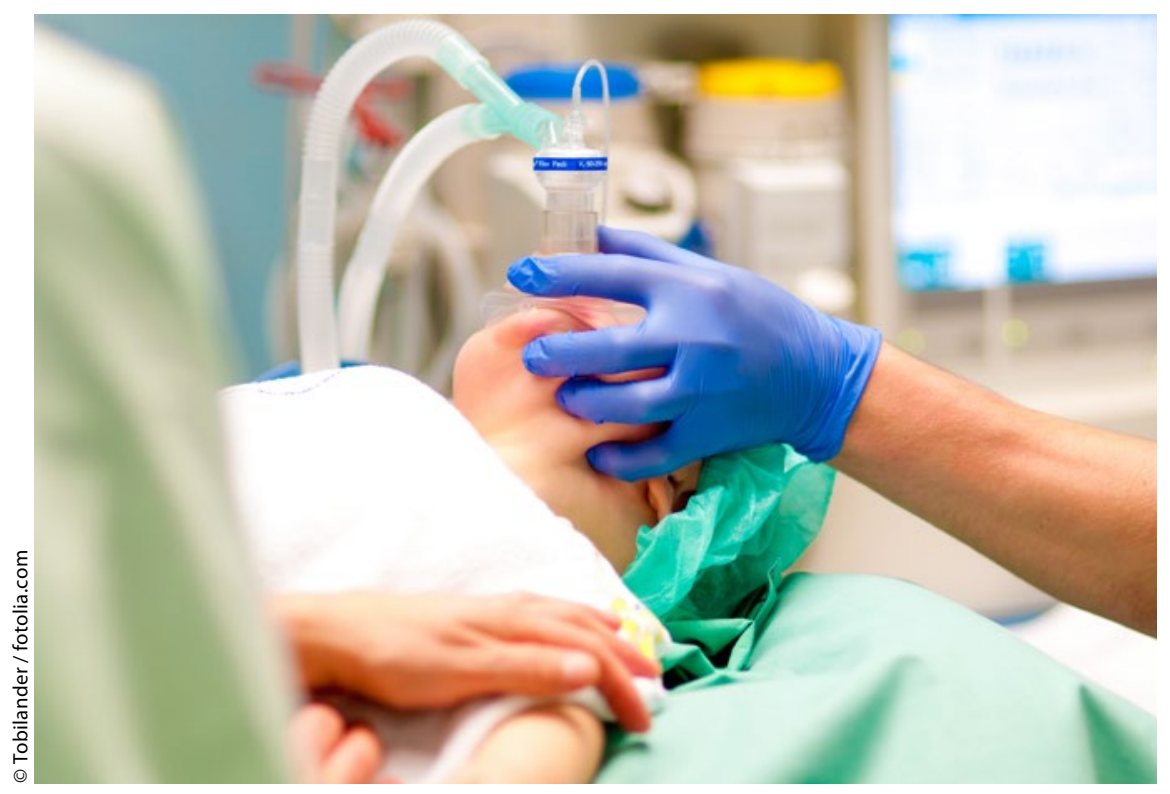

Aktuellen Studienergebnissen zufolge könnte eine Allgemeinästhesie den Nutzen der Thrombektomie gefährden.

$\mathrm{D}$ ie MRCLEAN-Studie war die erste Arbeit, die die Überlegenheit einer Schlaganfallbehandlung durch die sequenzielle Kombination aus intravenöser Thrombolyse und Thrombektomie im Vergleich zur reinen Lysetherapie bei Verschluss einer proximalen hirnversorgenden Arterie nachweisen konnte. Mehrere Studien folgten mit ähnlichem Ergebnis.

Berkhemer und Kollegen publizierten nun eine Post-hoc-Analyse der MRCLEAN-Studiendaten bezüglich des Behandlungserfolgs in Abhängigkeit von der Wahl des Anästhesieverfahrens. Dabei wurde die Gruppe mit Allgemeinanästhesie (einschließlich Intubation oder Anwendung einer Larynxmaske; 79 Patienten) mit der Gruppe ohne Narkose (137 Patienten) verglichen.

Die Patienten mit Thrombektomie ohne Allgemeinanästhesie zeigten drei Monate nach der Behandlung einen deutlichen Benefit im Vergleich zur reiein gutes neurologisches Ergebnis (mRS 0-2, d.h. funktionelle Unabhängigkeit)

Bei den Betroffenen, die eine Narkose erhielten, lag der Unterschied zur Gruppe der Patienten ohne Thrombektomie nur noch bei $4 \%$ und war damit nicht (!) mehr signifikant.

Auch in anderen Endpunkte hatten die nicht narkotisierten Patienten erhebliche Vorteile, so z.B. für den NIHSS nach 24 Stunden und sieben Tagen, für die Rekanalisationsrate und das finale Infarktvolumen.

Berkhemer OA et al. The effect of anesthetic management during intra-arterial therapy for acute stroke in MR CLEAN. Neurology. 2016; 87(7):656-664

\section{Kommentar}

Seit Anfang 2015 ist aufgrund von MRCLEAN und mehreren Folgestudien die Thromb- nen Lyse. So erhöhte sich die Chance auf absolut um $19 \%$. ektomie neben der intravenösen Thrombolyse eine etablierte zusätzliche Behandlung des Schlaganfalls bei proximalem Gefäßverschluss. Mehrere retrospektive Auswertungen legen jedoch nahe, dass der Erfolg dieser Behandlung offenbar von der Wahl des Anästhesieverfahrens abhängt. Die Publikation von Berkhemer und Kollegen reiht sich in diese Riege von Analysen ein. Für MRCLEAN heißt das konkret: Patienten, die eine zusätzliche Thrombektomie in Allgemeinanästhesie erhielten, profitierten von diesem Eingriff hinsichtlich ihres neurologischen Ergebnisses nicht, was hingegen bei den nicht narkotisierten Patienten sehr wohl der Fall war.

Die Autoren erklären den doch "dramatischen" Unterschied zwischen beiden Gruppen durch mehrere Faktoren, u. a. die längere Dauer bis zur Gefäßpunktion, die Induktion eines Blutdruckabfalls unter Narkoseeinleitung, Veränderungen der zerebrovaskulären Autoregulation und mögliche Komplikationen durch die Intubation selbst. Ist nach den vorliegenden Daten von einer Thrombektomie in Allgemeinanästhesie grundsätzlich abzuraten? Jein! Drei Studien (ANSTROKE, SIESTA und GOLIATH) untersuchen derzeit den Einfluss des Anästhesieverfahrens auf den Erfolg einer kombinierten Lyse und Thrombektomie mit prospektivem Ansatz. Dabei konnten die SIESTA-Initiatoren der Uniklinik Heidelberg kürzlich auf dem Jahreskongress der European Stroke Organisation erste Ergebnisse präsentieren. Hier zeigte sich zwischen den Patienten mit und ohne Allgemeinanästhesie kein nachweisbarer Unterschied zumindest für den NIHSS 24 Stunden nach dem Schlaganfallereignis (www.eso-stroke.org/fileadmin/files/2016/ eso/ESOC_News/ESOC_2016_Day_Two_ Highlights_May_11.pdf).

Die weiteren Daten aus SIESTA und den anderen Studien bleiben abzuwarten. Mit diesen wird hoffentlich eine abschließende Beurteilung dieser Thematik, einschließlich einer konsentierten Leitlinienempfehlung möglich werden.

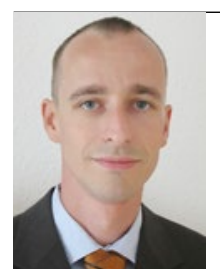

Dr. med. Sven Lerch Abteilung Neurologie, Bundeswehrkrankenhaus Berlin 